\title{
The effect of alcohol and placebo on post-error adjustments
}

\author{
Klaas Bombeke ${ }^{\dagger}$, Nathalie Schouppe ${ }^{\dagger}$, Wout Duthoo and Wim Notebaert *
}

Department of Experimental Psychology, Ghent University, Ghent, Belgium

Edited by:

Srikantan S. Nagarajan, University of California, San Francisco, USA

Reviewed by:

Marc Himmelbach, University

Hospital Tuebingen, Germany

Lluís Fuentemilla, University of

Barcelona, Spain

\section{*Correspondence:}

Wim Notebaert, Department of Experimental Psychology, Ghent University, Henri Dunantlaan 2, 9000 Ghent, Belgium.

e-mail: wim.notebaert@ugent.be

tThese authors equally contributed to this work.
Several studies have shown detrimental effects of alcohol on post-error adjustments. In contrast to previous studies, which focused on only one aspect of post-error adaptive behavior, we compared the effect of alcohol and placebo on post-error slowing (PES), post-error reduction of interference (PERI) and post-error improvement of accuracy (PIA). Moreover, we used a between-subjects design $(N=45)$ comparing a control condition to both an alcohol and an alcohol-placebo condition as to disentangle physiological and expectancy effects of alcohol. In a standard Stroop congruency task, we found that intoxicated participants as well as participants with the incorrect belief of being intoxicated showed significant decreased PES compared to a control group. Furthermore, we found evidence for a condition-independent post-error increase of interference and post-error decrease of accuracy. The underlying mechanisms of the post-error adaptation effects are discussed in terms of the orienting account (Notebaert et al., 2009).

Keywords: alcohol, placebo, post-error slowing, post-error reduction of interference, post-error improvement of accuracy, orienting account

\section{INTRODUCTION}

Cognitive control is the ability to detect difficulties and errors and to adjust our information processing system in order to optimize future action outcomes. A meta-analysis of Steele and Southwick (1985) suggests that alcohol typically impairs performance on tasks that involve cognitive control. More specifically, they demonstrated that alcohol has a detrimental effect in situations with simultaneous activation of conflicting responses (i.e., response conflict).

In the lab, conflict processing is typically investigated using congruency tasks, where irrelevant information may or may not interfere with response selection. In a Stroop task, for instance, participants have to respond on the basis of the color of a colorword, while the meaning of the word is irrelevant (Stroop, 1935). Participants are generally faster and more accurate on congruent (word GREEN in green ink) than on incongruent trials (word RED in green ink). Importantly, Curtin and Fairchild (2003) observed that, compared to a control group, intoxicated participants were slower and less accurate on incongruent trials, but not on congruent trials, suggesting impaired conflict processing for intoxicated participants.

Conflict processing, which takes place on incongruent trials, has been localized in posterior mediofrontal cortex (pMFC). One influential theory holds that pMFC monitors for the occurrence of conflict, after which the need for cognitive control is signaled (Botvinick et al., 2001). A role for pMFC in conflict detection has been confirmed by a large number of brain imaging studies, showing increased pMFC activity on incongruent trials (e.g., Kerns et al., 2004; Egner and Hirsch, 2005; Carter and van Veen, 2007). Interestingly, alcohol intake led to decreased pMFC activity on incongruent trials (Marinkovic and Azma, 2010; Marinkovic et al., 2011), explaining the selective deficit for incongruent trials.
More important for the present study, the pMFC is also involved in the detection of errors and subsequent behavioral adjustments. Several studies have reported a correlation between pMFC activity and post-error adaptations (Gehring et al., 1993; Kerns et al., 2004; Wessel and Ullsperger, 2011). Interestingly, alcohol seems to decrease error-related activity in pMFC: the error-related negativity (ERN), an electrophysiological component related to pMFC activation (Gehring et al., 1993), was found to be reduced after alcohol consumption (Ridderinkhof et al., 2002; Bartholow et al., 2011). Taken together, these findings suggest that alcohol would also yield impaired post-error adaptation effects. Surprisingly, this has not been tested systematically.

In the error literature, three post-error adaptation effects have been reported for which different theoretical accounts have been described (for a review, see Danielmeier and Ullsperger, 2011). First, post-error slowing (PES; Rabbitt, 1966) refers to the finding that participants typically slow down after making an error. It has been suggested that PES reflects a strategy shift where participants deliberately slow down in order to decrease error likelihood in following trials (e.g., Botvinick et al., 2001). According to this account, errors will also be detected by the conflict monitoring system, in turn triggering enhanced attentional control. Alternatively, the orienting account by Notebaert et al. (2009) frames PES as an orienting reaction to infrequent errors, slowing down the following response. In line with this view, Notebaert et al. (2009) predicted that slowing would occur after an infrequent event, irrespective of the nature of that event (incorrect or correct answer). Accordingly, their results showed PES in the condition where errors were low in frequency, and post-correct slowing in the condition where errors were high frequent (see also Houtman et al., 2011). Second, it has been shown that participants' congruency effects are smaller following an error 
(post-error reduction of interference, PERI; Ridderinkhof et al., 2002). This effect is explained by the cognitive control account as reflecting increased attentional focusing after an erroneous response. However, the orienting account does not predict such effect, since it is presumed that attention is directed away from the task after an error. The orienting account therefore predicts an inverse PERI effect. Finally, post-error increase in accuracy (PIA) is the finding that accuracies are higher after errors than after correct trials (e.g., Laming, 1968; Marco-Pallarés et al., 2008; Danielmeier et al., 2011; Seifert et al., 2011). PIA is predicted by the cognitive control account: control is increased after an error and therefore fewer errors are committed. However, some studies have failed to find increased accuracy following errors (e.g., Hajcak et al., 2003; Hajcak and Simons, 2008; King et al., 2010) or even observed a post-error accuracy decrease (e.g., Rabbitt and Rodgers, 1977; Fiehler et al., 2005), which is more in line with the orienting account.

To date, only two studies reported the effect of alcohol on posterror behavioral adaptations, focusing only on the PERI effect. These studies showed a smaller PERI effect in an alcohol group, compared to a placebo group (Ridderinkhof et al., 2002) and a control group (Bartholow et al., 2011). No study examined alcohol effects on PES or PIA. The goal of our study was therefore to investigate the effect of alcohol on error adaptation more thoroughly. Unlike Ridderinkhof et al. (2002) and Bartholow et al. (2011), who only looked at PERI, we wanted to compare the effect of alcohol on PES, PERI, and PIA by making use of a standard congruency task. The fact that these three effects can be analyzed in one and the same task is an ideal situation to obtain knowledge about the specific ways alcohol affects cognitive processing.

We used a design that enabled us to separate the physiological and psychological effects of alcohol. The balanced placebo design (Marlatt and Rohsenow, 1980) is a $2 \times 2$ design consisting of two dichotomous factors: beverage manipulation and correctness of information. The beverage manipulation factor determined whether a participant had to drink an alcoholic or a non-alcoholic beverage. Correctness of information determined whether the person was correctly informed about his drinks or not (right or wrong). For ethical reasons, we did not incorporate an alcohol group that was told that they were not drinking alcohol. The alcohol-placebo condition, in which participants believed they were drinking alcohol but were in fact not, is enough to dissociate physiological from psychological effects.

\section{MATERIALS AND METHODS PARTICIPANTS}

Forty-five healthy male participants (18-38 years old, $M=20.84$, $S D=3.23)$ participated in this study. They were non-smokers and did not use medication or drugs. All participants had a normal or corrected-to-normal vision. In order to ensure the alcohol would have a similar effect on all participants, we only used "social drinkers" who drink on average between the 1.8 and 3.5 standard drinks a day (or 12.6-24.5 a week). Participants were paid for their participation and gave their permission prior to each experimental session by filling in informed consents approved by the ethical committee.

\section{DESIGN}

The double-blind balanced placebo design of Marlatt and Rohsenow (1980) with three groups was used. All participants were randomly distributed over the groups. The first condition $(N=15)$ was the alcohol group $(M=19.53$ years, $S D=1.25$ years). They had to drink alcohol and were correctly informed. The second condition ( $N=15)$ was the control group $(M=22.8$ years, $S D=4.52$ years). They had to drink a beverage without alcohol and were also correctly informed. The third condition $(N=15)$ was the alcohol-placebo group $(M=20.2$ years, $S D=$ 2.08 years). They were informed that they were receiving alcohol but in reality the beverage contained no alcohol.

\section{PROCEDURE}

\section{Consent and screening}

Participants were asked not to use drugs or alcohol $24 \mathrm{~h}$ before the start of the experiment. They were also asked to abstain from drinking and eating $4 \mathrm{~h}$ in advance. The experiment always started at 13 or $15 \mathrm{~h}$ in the afternoon to avoid effects of the circadian rhythm. They were also asked to sign a document in which they gave their permission to stay at the faculty until their intoxication level was lower than the legally permitted level. In their first session, participants also had to fill in two questionnaires regarding their average alcohol consumption in daily life: the AUDIT (Saunders et al., 1993) and the Timeline follow-back questionnaire (Sobell and Sobell, 1992).

\section{Beverage manipulation}

The alcohol manipulation was based on Ridderinkhof et al. (2002). In the alcohol condition, participants had to drink two cups containing $0.55 \mathrm{~g}$ alcohol in total. Dependent on parameters like weight and alcohol tolerance, an intake of $0.55 \mathrm{~g} / \mathrm{kg}$ alcohol results in a blood-alcohol concentration (BAC) of $80 \mathrm{mg} / 100 \mathrm{ml}$ $(=0.7-0.8 \%$ o $)$. By means of the formula of Widmark (Watson et al., 1981), it was determined how much vodka was needed in order to achieve a BAC of $80 \mathrm{mg} / 100 \mathrm{ml}$. This formula predicts the BAC with a number of parameters (for the exact formula, see Watson et al., 1981). We chose vodka because of its high alcohol concentration $(37.5 \%$ alcohol), making it unnecessary to use more than two cups (in contradiction to beer or wine). The vodka was equally distributed in two identical cups. In each cup, green peppermint syrup (with a quantity of $75 \%$ of the amount of vodka) was added. This peppermint syrup made it impossible for the participants to taste the presence of alcohol in the cups. Furthermore, the cups were supplemented with orange juice until each cup contained $400 \mathrm{ml}$ of liquid. A cover was put on each cup and participants had to drink the cocktail with a reed. In the placebo condition, the participant had to drink two $400 \mathrm{ml}$ cups which contained only orange juice and mint syrup. The calculation of the amount of peppermint syrup was exactly the same as in the alcohol session. Thus, the only difference between the alcohol and placebo conditions was the presence of vodka. Participants' BAC was measured at their arrival to verify that they were completely sober and started the experiment with a BAC of zero. The drinking part of each experimental session consisted of three phases. The first $20 \mathrm{~min}$, participants had to drink the first cup of $400 \mathrm{ml}$. They were asked to drink at a regular rate. The 
next $20 \mathrm{~min}$, they had to drink the second cup of $400 \mathrm{ml}$. Finally, there was a waiting period of $20 \mathrm{~min}$ in order to give the alcohol the time to spread in the blood and have its physiological effect. The second BAC measurement was right before the start of the task. Furthermore, there were also measurements after every two blocks of the experimental task.

\section{Task}

Stimuli were presented on a 17 -inch monitor with a distance of $50 \mathrm{~cm}$ between the screen and the participant. The experiment was programmed with T-scope software (Stevens et al., 2006). Stroop stimuli consisted of four color words (RED, GREEN, BLUE, or YELLOW), presented in red, green, blue, or yellow ink. Participants were instructed to respond to the ink color of the stimulus by pressing an associated button of a response box. Response mapping of colors was counterbalanced across participants. The instructions at the beginning of the experiment highlighted the importance of memorizing the response mapping and these instructions were repeated between every block. A trial started with the presentation of a fixation cross for $500 \mathrm{~ms}$. Next, a stimulus word appeared and the participant had to react as fast and accurately as possible. The maximum response time was limited to $1500 \mathrm{~ms}$. In order to avoid people forgetting the response mapping, feedback was given after every trial. A correct response was followed by the letter "J," an incorrect response by the letter "F" and a too slow response by the letter "T." The meaning of these letters was also explained in the instructions. Participants had to perform 10 blocks of 100 trials each. The first block was considered a practice block and would not be included in the analyses afterward. Each block was randomized and consisted of 50 congruent trials and 50 incongruent trials.

\section{RESULTS}

\section{QUESTIONNAIRES}

The purpose of the questionnaires was to check if the three beverage conditions did not differ from each other with regard to alcohol usage in daily life. The three conditions did not significantly differ on the AUDIT (Saunders et al., 1993), $F_{(2,42)}<1$, and the Timeline follow-back questionnaire (Sobell and Sobell, 1992), $F_{(2,42)}<1$.

\section{ALCOHOL MANIPULATION}

Because this experiment has a between-subjects design, the following analyses only concern the 15 persons in the alcohol group. At their arrival at the lab, all participants were completely sober (BAC $=0 \mathrm{mg} / 100 \mathrm{ml}$ ). Just before the start of the task, the average $\mathrm{BAC}$ was $M=92.6(S D=20.5) \mathrm{mg} / 100 \mathrm{ml}$. After two, four, six, eight, and ten blocks, it was respectively $M=98.7$ ( $S D=$ 19.5), $M=101.8(S D=19.2), M=101.9(S D=20.5), M=$ $100.7(S D=21.3)$, and $M=99.5(S D=20) \mathrm{mg} / 100 \mathrm{ml}$. One hour after they finished the task, the alcohol concentration of most participants fluctuated around $0.5 \%$.

In the post-questionnaire, all participants in the alcohol group indicated they were sure that they were drinking alcohol. In the control group, all participants were sure they were not drinking alcohol. Most importantly, all 15 participants in the alcoholplacebo group seemed to have believed they had been drinking an alcoholic beverage. This showed that the manipulation was successful and we could make valid conclusions about the different conditions.

\section{POST-ERROR ADJUSTMENTS}

The practice block and the first trial of each experimental block were removed. Next, responses that exceeded the maximum response limit $(1.4 \%)$ and incorrect responses were excluded $(8.4 \%)$. An outlier removal criterion of two SDs, calculated per participant and per congruency, was used (removal of $4.86 \%$ ). The data were aggregated on the mean.

\section{RT}

A repeated-measures ANOVA with the within-subject variables previous accuracy (correct or incorrect) and current congruency (congruent or incongruent) and the between-subject variable beverage condition (alcohol, placebo, or control) was conducted on mean RTs. Two participants showed a low number of observations (i.e., <5) in one cell of the repeated-measures ANOVA for this analysis. However, excluding these two participants did not change the overall pattern of results. The results based on all participants are reported.

The main effect of current congruency was significant, $F_{(1,42)}=101.96, p<0.001, r=0.84$, indicating that people were faster on congruent trials $(578 \mathrm{~ms})$ than on incongruent trials $(644 \mathrm{~ms})$. The interaction between the congruency effect and condition was not significant, $F_{(2,42)}=1.39, p>0.1, r=0.25$. The main effect of condition was also not significant, $F_{(2,42)}<1$, $p>0.1, r=0.18$.

PES. The main effect of previous accuracy was significant, $F_{(1,42)}=68.81, p<0.001, r=0.79$, indicating that participants were slower after an error than after a correct response (i.e., PES; see Figure 1). Furthermore, the interaction between previous accuracy and condition also turned out significant, $F_{(2,42)}=$ $3.89, p<0.05, r=0.39$, indicating that PES differed significantly between the conditions, while PES was significant in all conditions (all $p s<0.001$ ). Contrast analyses revealed that the control group showed a significantly larger PES effect (79.52 ms) than the

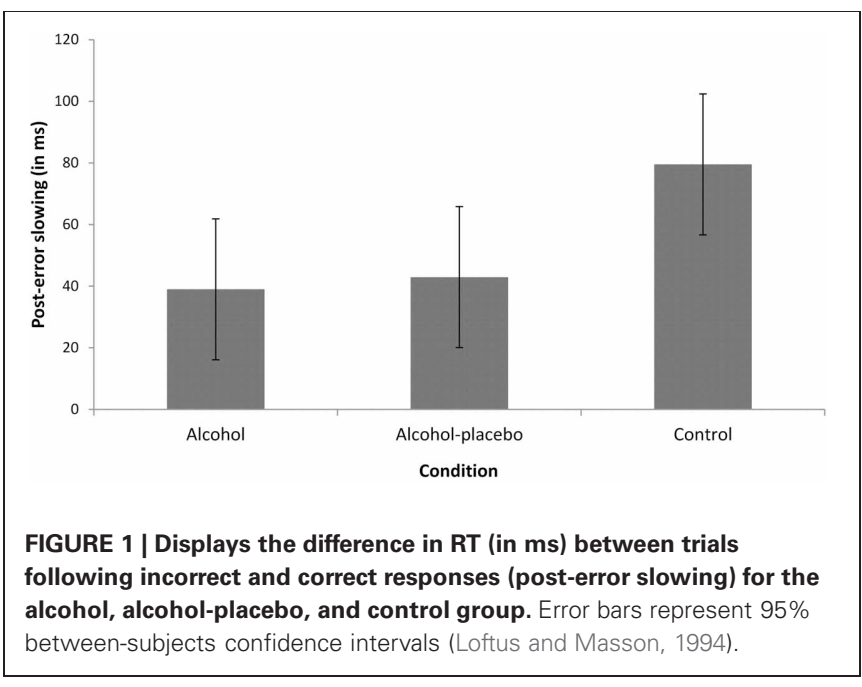




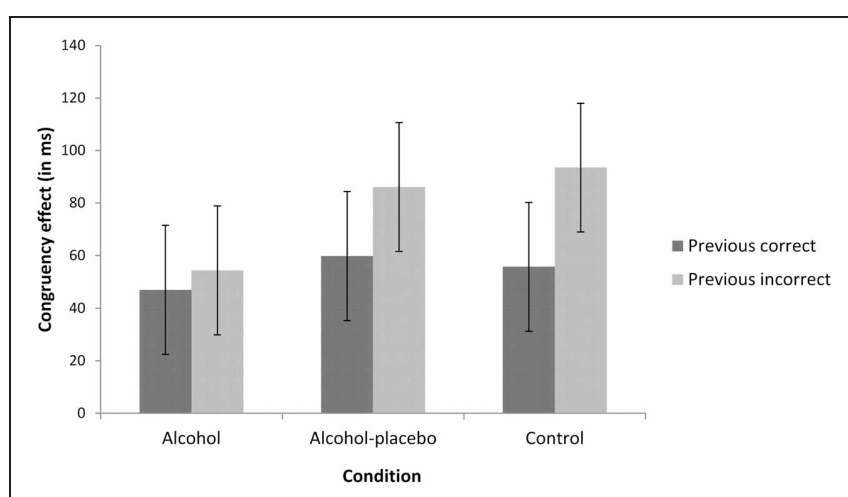

FIGURE 2 | Displays the congruency effect (incongruent-congruent) in ms for trials following correct and incorrect responses for the alcohol, alcohol-placebo, and control group. Error bars represent 95\% within-subjects confidence intervals based on the mean square error term of the interaction between previous accuracy and condition (Loftus and Masson, 1994)

alcohol group (38.97 ms), $F_{(1,28)}=6.92, p<0.05, r=0.44$, and a marginally significant larger PES effect than the alcohol-placebo group (42.93 ms), $F_{(1,28)}=3.95, p=0.057, r=0.35$. There was no difference between the alcohol group and the alcohol-placebo group, $F_{(1,28)}<1$.

PERI. We found an interaction between previous accuracy and current congruency, $F_{(1,42)}=5.76, p<0.05, r=0.35$. As depicted in Figure 2, the congruency effect was larger following errors $(77.97 \mathrm{~ms})$ than following correct trials $(54.15 \mathrm{~ms}$; i.e., post-error increase of interference). This reversed PERI effect did not interact with condition, $F_{(2,42)}<1, p>0.1, r=0.19$.

\section{Accuracy}

A repeated measures ANOVA with the within-subject variables previous accuracy and current congruency and the betweensubject variable condition was conducted on mean error rates. Overall, the error rate was on average $8.5 \%(S D=5.7 \%)$. No main effect of condition was found, $F_{(2,42)}<1, p>0.1, r=$ 0.15 , indicating that the differences in PES across conditions were not due to different error frequencies. A main effect of current congruency was found, $F_{(1,42)}=11.72, p<0.001, r=0.47$, showing that participants were more accurate on congruent trials $(7.1 \%)$ than on incongruent trials $(8.4 \%)$. The interaction between current congruency and condition was not significant, $F_{(2,42)}<1, p>0.1, r=0.13$.

PIA. The main effect of previous accuracy was significant, $F_{(1,42)}=11.73, p<0.001, r=0.47$, indicating that participants were less accurate after an error (12.21\%) than after a correct response $(7.97 \%$, see Figure 3$)$. The interaction between previous accuracy and condition was not significant, $F_{(2,42)}=1.20$, $p>0.1, r=0.23$.

PERI. The interaction between previous accuracy and congruency turned out marginally significant, $F_{(1,42)}=3.16, p=0.083$, $r=0.26$, indicating a larger congruency effect after an error than after a correct response. There was again no interaction between

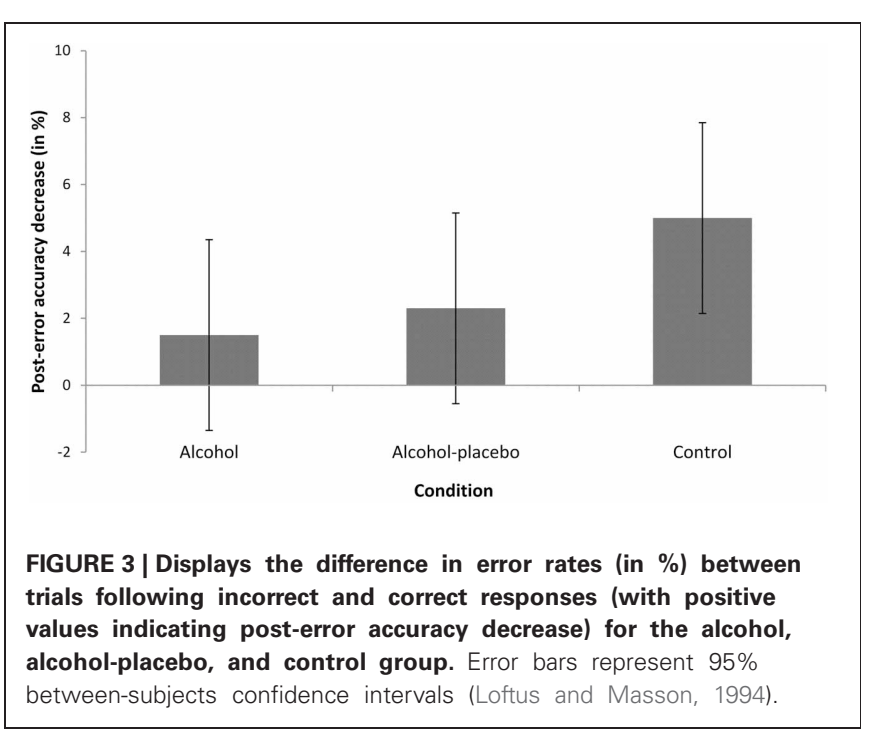

previous accuracy, congruency and condition, $F_{(2,42)}=1.68$, $p \geq 0.1, r=0.27$.

\section{DISCUSSION}

The first goal of our study was to perform a more comprehensive investigation of the effects of alcohol on post-error behavioral adaptation. Interestingly, results showed a significant condition-dependent PES effect: PES was smaller in both the alcohol and alcohol-placebo group than in the control group. Furthermore, contrary to the results of Ridderinkhof et al. (2002) and Bartholow et al. (2011) reporting a condition-dependent PERI effect, we observed a reversed PERI effect (i.e., a larger congruency effect following errors) that did not differ between groups. Similarly, we observed decreased accuracy following errors, independent of condition.

\section{PES}

The observation that PES decreased in both the alcohol and the alcohol-placebo group supports an explanation in terms of expectancy effects: the performance of someone who beliefs to be intoxicated resembles the performance of someone who is actually intoxicated. It is important to note that the error rates in the different alcohol conditions did not differ significantly. If this would have been the case, the condition-dependent PES effect could be the result of the negative correlation between error rates and PES: the higher the error rate, the lower PES (Houtman et al., 2011). However, it appears that alcohol expectancy reduces the saliency of errors, perhaps because one expects to make more errors. This can be interpreted within the orienting account, which predicts that slowing will occur after an infrequent event, irrespectively of the nature of that event. The expectation to commit more errors intoxicated or under the impression of being intoxicated seems to have the effect that an error is no longer perceived as a surprising event.

\section{PERI/PIA}

Whereas a cognitive control account would assume an increased focus on the task following errors, reflected in a reduced 
congruency effect, we found an enhanced congruency effect on post-error trials. This finding seems more consistent with the orienting account (Notebaert et al., 2009), which argues that errors or error-related processes interfere with the task at hand.

In line with this prediction, we observed reduced accuracy following errors, as was also observed in Rabbitt and Rodgers (1977) and Fiehler et al. (2005). Desmet et al. (2012), however, recently demonstrated that in a more complex, mental arithmetic task, PIA can be observed.

Although the orienting account can capture decreased accuracy and increased congruency effects after errors, it would also predict an interaction with alcohol group, in the sense that both effects should depend on error expectancy. The fact that both effects are independent of alcohol condition, suggests that increased error rates and congruency effects following errors are unrelated to the mechanism(s) responsible for PES. Such dissociation between the three post-error adaptation effects has already been reported in the literature. For instance, De Bruijn et al. (2004) demonstrated that lorezepam, a $\mathrm{GABA}_{\mathrm{A}}$-inducing drug, had a selective negative effect on PERI, but not on PES. Recently, King et al. (2010) also

\section{REFERENCES}

Bartholow, B. D., Erika, A. H., Lust, S. A., Saults, J. S., and Wood, P. K. (2011). Alcohol effects on performance monitoring and adjustment: affect modulation and impairment of evaluative cognitive control. J. Abnorm. Psychol. 121, 173-186.

Botvinick, M. M., Braver, T. S., Barch, D. M., Carter, C. S., and Cohen, J. D. (2001). Conflict monitoring and cognitive control. Psychol. Rev. 108, 624-652.

Carter, C. S., and van Veen, V. (2007). Anterior cingulate cortex and conflict detection: an update of theory and data. Cogn. Affect. Behav. Neurosci. 7, 367-379.

Curtin, J. J., and Fairchild, B. A. (2003). Alcohol and cognitive control: implications for regulation of behavior during response conflict. J. Abnorm. Psychol. 112, 424-436.

Danielmeier, C., Eichele, T., Forstmann, B. U., Tittgemeyer, M., and Ullsperger, M. (2011). Posterior medial frontal cortex activity predicts post-error adaptations in task-related visual and motor areas. J. Neurosci. 31, 1780-1789.

Danielmeier, C., and Ullsperger, M. (2011). Post-error adjustments. Front. Psychology 2:233. doi: 10.3389/fpsyg.2011.00233

De Bruijn, E. R. A., Hulstijn, W., Verkes, R. J., Ruigt, G. S. F., and Sabbe, B. G. C. (2004). Drug-induced simulation and suppression of action monitoring in healthy volunteers.
Psychopharmacology

177 151-160.

Desmet, C., Imbo, I., and De Brauwer, J. (2012). Error adaptation in mental arithmetic. Q. J. Exp. Psychol. 65, 1059-1067.

Egner, T., and Hirsch, J. (2005). Cognitive control mechanisms resolve conflict through cortical amplification of task-relevant information. Nat. Neurosci. 8, 1784-1790.

Fiehler, K., Ullsperger, M., and Von Cramon, D. Y. (2005). Electrophysiological correlates of error correction. Psychophysiology $42,72-82$.

Gehring, W. J., Goss, B., Coles, M. G. H., Meyer, D. E., and Donchin, E. (1993). A neural system for error-detection and compensation. Psychol. Sci. 4, 385-390.

Hajcak, G., McDonald, N., and Simons, R. F. (2003). To err is autonomic: error-related brain potentials, ANS activity, and post-error compensatory behavior. Psychophysiology 40, 895-903.

Hajcak, G., and Simons, R. F. (2008). Oops!.. I did it again: an ERP and behavioral study of double-errors. Brain Cogn. 68, 15-21.

Houtman, F., Núñez Castellar, E., and Notebaert, W. (2011). Orienting to errors with and without immediate feedback. J. Cogn. Psychol. 24, 278-285.

Kerns, J. G., Cohen, J. D., MacDonald, A. W., Cho, R. Y., Stenger, V. A., and Carter, C. S. (2004). Anterior

showed that PES and PERI are mediated by different neural structures.

Taken together, our data allow for two important conclusions. First, alcohol and alcohol expectancy decrease PES. Although it is theoretically possible that this reduction in PES is caused by different factors (one physiological and one psychological, for instance), the most parsimonious explanation is that both groups show reduced PES because they both expect reduced performance, and hence increased error rates. Several recent studies have indicated that increased error rates decrease PES, presumably because errors become less salient when more errors are made (Houtman et al., 2011). Following the same logic, we argue that the expectation of making more errors also reduces the saliency of errors. Second, our data support previous studies that concluded that PES, PERI, and PIA are not caused by one and the same mechanism (e.g., King et al., 2010), as alcohol and alcohol expectancy only influences PES and not PERI or PIA.

\section{ACKNOWLEDGMENTS}

Nathalie Schouppe is sponsored by FWO 3F011209, Wout Duthoo by FWO 3F009109 and Wim Notebaert by FWO G.0769.11N and BOF 01J11409.

cingulate conflict monitoring and adjustments in control. Science 303 , 1023-1026.

King, J. A., Korb, F. M., von Cramon, D. Y., and Ullsperger, M. (2010). Post-error behavioral adjustments are facilitated by activation and suppression of task-relevant and task-irrelevant information processing. J. Neurosci. 30, 12759-12769.

Laming, D. R. J. (1968). Information Theory of Choice-reaction Times. New York, NY: Academic Press.

Loftus, G. R., and Masson, M. E. J. (1994). Using confidence intervals in within-subject designs. Psychon. Bull. Rev. 1, 476-490.

Marco-Pallarés, J., Estela, C., Münte, T. F., and Rodríguez-Fornells, A. (2008). Neural mechanisms underlying adaptive actions after slips. J. Cogn. Neurosci. 20, 1595-1610.

Marinkovic, K., and Azma, S. (2010). Neural dynamics of alcohol effects on cognitive control: Eriksen flanker task. IFMBE Proc. 28, 374-377.

Marinkovic, K., Rickenbacher, E. Azma, S., and Artsy, E. (2011). Acute alcohol intoxication impairs top-down regulation of Stroop incongruity as revealed by blood oxygen level-dependent functional magnetic resonance imaging. Hum. Brain Mapp. 333, 319-333.

Marlatt, G. A., and Rohsenow, D. J. (1980). "Cognitive processes in alcohol use: expectancy and the balanced placebo design," in Advances in Substance Abuse: Behavioral and Biological Research, 1st Edn., ed N. K. Mello (Greenwich, CT: JAI Press), 159-199.

Notebaert, W., Houtman, F., Van Opstal, F., Gevers, W., Fias, W., and Verguts, T. (2009). Post-error slowing: an orienting account. Cognition 111, 275-279.

Rabbitt, P., and Rodgers, B. (1977). What does a man do after he makes an error? An analysis of response programming. Q. J. Exp. Psychol. 29, 727-743.

Rabbitt, P. M. A. (1966). Errors and error correction in choice reaction tasks. J. Exp. Psychol. 71, 264-272.

Ridderinkhof, K. R., de Vlugt, Y., Bramlage, A., Spaan, M., Elton, M., Snel, J., et al. (2002). Alcohol consumption impairs detection of performance errors in mediofrontal cortex. Science 298, 2209-2211.

Saunders, J. B., Aasland, O. G., Babor, T. F., De La Fuente, J. R., and Grant, M. (1993). Development of the alcohol use disorders identification test (AUDIT): WHO collaborative project on early detection of persons with harmful alcohol consumption-II. Addiction 88, 791-804.

Seifert, S., Von Cramon, D. Y., Imperati, D., Tittgemeyer, M., and Ullsperger, M. (2011). Thalamocingulate interactions in performance monitoring. J. Neurosci. 31, 3375-3383. 
Sobell, L. C., and Sobell, M. B. (1992). "Timeline follow-back: a technique for assessing self-reported alcohol consumption," in Measuring Alcohol Consumption: Psychosocial and Biochemical Methods, eds R. Z. Litten and J. P. Allen (Totowa, NJ: Humana Press), 41-72.

Steele, C. M., and Southwick, L. (1985). Alcohol and social behavior I: the psychology of drunken excess. J. Pers. Soc. Psychol. 48, 18-34.

Stevens, M., Lammertyn, J., Verbruggen, F., and Vandierendonck, A. (2006). Tscope: a C library for programming cognitive experiments on the MS Windows platform. Behav. Res. Methods 38, 280-286.

Stroop, J. R. (1935). Studies of interference in serial verbal reactions. J. Exp. Psychol. 18, 643-662.

Watson, P. E., Watson, I. D., and Batt, R. D. (1981). Prediction of blood alcohol concentrations in human subjects. Updating the Widmark equation. J. Stud. Alcohol 42, 547-556.

Wessel, J. R., and Ullsperger, M. (2011). Selection of independent components representing event-related brain potentials: a data-driven approach for greater objectivity. Neuroimage 54, 2105-2115.

Conflict of Interest Statement: The authors declare that the research was conducted in the absence of any commercial or financial relationships that could be construed as a potential conflict of interest.

Received: 09 October 2012; accepted: 05 January 2013; published online: 25 January 2013.
Citation: Bombeke K, Schouppe N, Duthoo W and Notebaert W (2013) The effect of alcohol and placebo on post-error adjustments. Front. Hum. Neurosci. 7:3. doi: 10.3389/fnhum.2013.00003

Copyright (C) 2013 Bombeke, Schouppe, Duthoo and Notebaert. This is an openaccess article distributed under the terms of the Creative Commons Attribution License, which permits use, distribution and reproduction in other forums, provided the original authors and source are credited and subject to any copyright notices concerning any third-party graphics etc. 